\title{
Male Infertility in Cryptozoospermia or Severe Oligozoospermia is sperm Useful Cryopreservation
}

\section{Aya AL-ibraheemi ${ }^{1}$, Mustafa Zakaria ${ }^{2 *}$, Mohamed Zarqaoui ${ }^{3}$, Mohamed Ennaji ${ }^{4}$, Wassym R Senhaji ${ }^{5}$, Ritu S Santwani ${ }^{6}$, Nisrine En-naciri ${ }^{7}$, Hafida Tarik ${ }^{7}$, Romaissa Boutiche ${ }^{8}$ and Noureddine Louanjli ${ }^{9}$}

${ }^{1}$ Embryologist, Graduated from University of Nottingham, United Kingdom, with a MMedSci Degree in Assisted Reproductive Technology and a Researcher in the Art Irifiv Scientific Research Group, UK

${ }^{2}$ Head of LABOMAC Laboratory of Clinical Analysis and Assisted Reproductive Technology IRIFIV Fertility Center, AFC Fertility Center - Executive Vice President of the ART IRIFIV Scientific Research Group (AISRG) Casablanca, Morocco ${ }^{3}$ Endoscopic Surgery Obstetrics and Gynecology FR, Coordinator of IRIFIV Fertility Center, Head of the ART IRIFIV Scientific Research Group (AISRG) Casablanca, Morocco ${ }^{4}$ Senior Clinical Embryologist at IRIFIV Fertility Center, Member of the Scientific Research Group and Researcher in the Scientific Research Group, Casablanca, Morocco ${ }^{5}$ Gynecologist and Obstetrician, Endoscopic Surgeon and Fertility Expert, $A$ Research in Art Irifiv Scientific Research Group Associated Practitioner at IRIFIV Fertility Center - Casablanca, Morocco

${ }^{6}$ Gynaecologist and Obstetrician, ART-Singapore, Honorary Professor, Obs and Genec - VIMS Medical College, Garjula - India Society for Assisted Reproduction (ISAR).is a researcher in the Art Irifiv Scientific Research Group, India ${ }^{7}$ Senior Clinical Embryologist, Laboratory IVF Agadir, Souss-Massa, Member of the Scientific Research Group and Researcher in the Scientific Research Group, Agadir, Morocco ${ }^{8}$ Senior Clinical Embryologist, Laboratory IVF Algeria, Rotaby Fertility Center Algiera - Algiers, Member of the Scientific Research Group and researcher in the Scientific Research Group, Algeria ${ }^{9}$ Reproductive Biology, and Assisted Reproductive Technology, Consultant at IRIFIV Fertility Center, Administrative Deputy and Writer for the ART IRIFIV Scientific Research Group (AISRG) Casablanca, Morocco

*Corresponding Author: Mustafa Zakaria, Consultant at IRIFIV Fertility Center, Administrative Deputy and Writer for the ART IRIFIV Scientific Research Group (AISRG), Casablanca, Morocco.
Received: January 15, 2021

Published: January 28, 2021

C All rights are reserved by Mustafa

Zakaria., et al. 


\section{Abstract}

Male infertility count for almost $30 \%$ of the total cases of infertility. Male infertility can be caused by several and various factors such as environmental, genetics and hormonal factors. Male infertility can be diagnosed through semen sample analysis; a fresh semen sample can indicate different semen abnormalities. A semen sample can indicate Azoospermia, Cryptozoospermia (Co) or Severe Oligozoospermia (ESO), who can cause infertility in male. It is challenging for an andrologist to distinguish between ESO and CO in clinic diagnose since both of them show no sperm in the sample in the initial sample test. In Cryptozoospermia or Severe Oligozoospermia, cryopreservation plays a significant part in preserving male fertility by freezing the individual sperm.

Keywords: Male Infertility; Semen Analysis; Abnormal Semen Sample; Cryptozoospermia and Cryopreservation

\section{Abbreviations}

ICSI: Intracytoplasmic Sperm Injection; APA: Advanced Paternal Age; TESA: Testicular Sperm Aspiration; PESA: Percutaneous Epididymal Sperm Aspiration; ZP: Zona Pellucida; CPA: Cryoprotective Agent; AZF: Azoospermia Factor; ESP: Extended Sperm Preparation; FSH: Follicle-stimulating Hormone; CO: Cryptozoospermia; ESO: Extremely Severe Oligozoospermia; LN2: Liquid Nitrogen.

\section{Introduction}

\section{Infertility}

Infertility is the failure to conceive or get pregnant within a sensible trying period, usually 6 to 12 months. Sterility is a complete failure to develop pregnancy, which can be reversible or irreversible. Subfertility is infertility without an absolute limit to reproduction which can cause sterility, such as azoospermia. Infertility can be categorised into three categories, female infertility, male infertility, and unknown factors infertility [1]. Female infertility counts for approximately 30\%, including anovulation, tubal obstruction, or other pathology, where male infertility disorder counts about $30 \%$, and conditions in both partners in 30\%. No abnormalities are found in approximately 10\% (Miyamoto., et al. 2012).

Male infertility

Male infertility is described as the incapability of a male to fertilize female oocytes and make a fertile female pregnant for at least one year of unprotected intercourse. Several factors can cause male infertility, such as genetics and environmental factors. Environmental factors can be produced by exposure to environmental toxins, obesity, and age (Leslie SW., et al. 2020), they below diagram illustrates most of the male infertility factors (Figure 1).

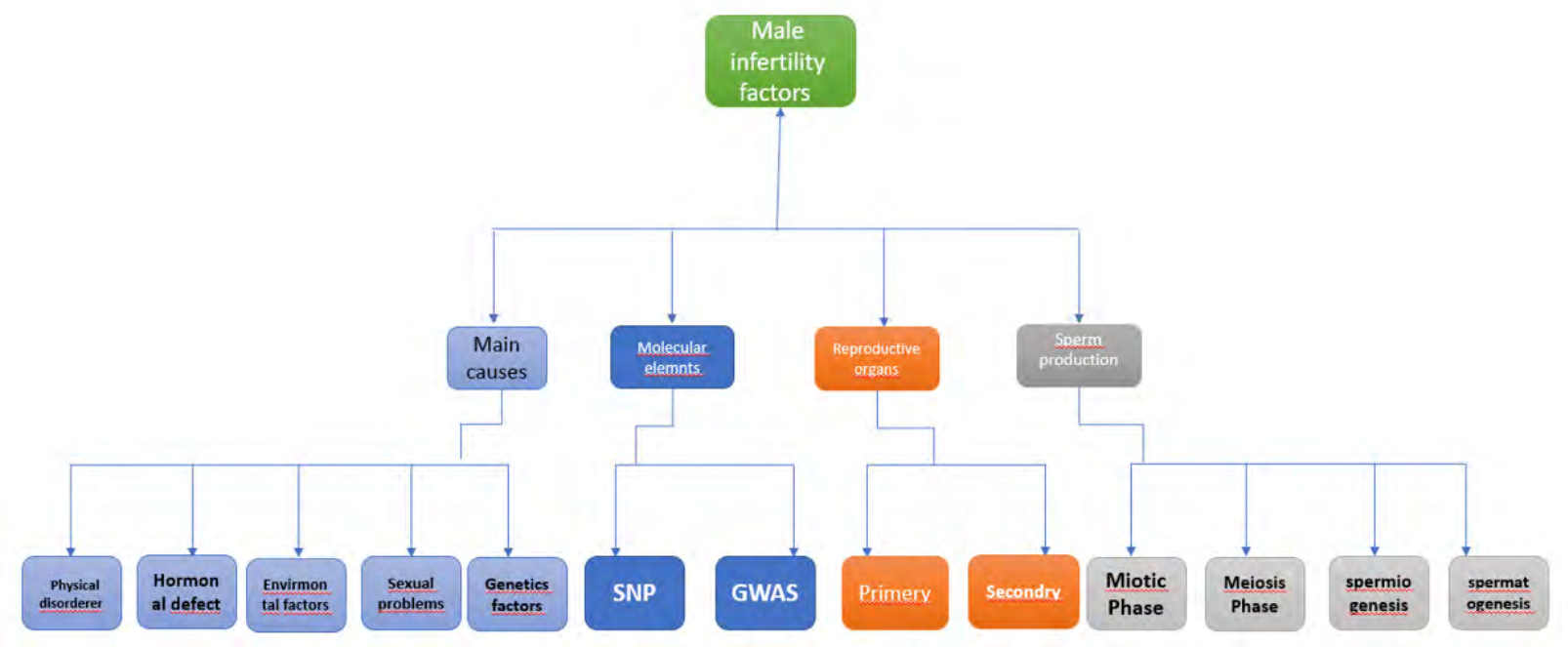

Figure 1: An illustrative flow-map of the main factors that can lead to male infertility (Babakhanzadeh., et al. 2012). 
The environmental factors association with male infertility has been studies and examined in several researches, where they have founded that men regularly are exposed to hazardous materials in their daily work such as solvents, pesticides, adhesives, silicones and radioactivity, exposure to these and related substances can be a potential cause of male infertility. Radiation exposure can reduce sperm production, and susceptibility to high dosages can cause complete infertility. Additionally, the overuse of the sunbath can likewise lead to a temporary drop in sperm count. Besides, jobs which need long-time of sitting (such as driving) or remaining exposed to high temperatures (such as bakeries) can negatively influence fertility. There is no faultless understanding of their sperm parameters and fertility outcomes regarding alcohol consumption and smoking. Nevertheless, increasing sperm degradation quality may be linked to cigarette smoking and alcohol consumption. Poor nutrition can also play a part in male infertility. Men are usually seen to be protected against age fertility problems. However, assisted reproductive technologies start to raised awareness about the advanced paternal age (APA) Besides, APA has also been linked with numerous offspring sicknesses like achondroplasia, autism, schizophrenia and bipolar disorders (Sharma., et al. 2015). Firstly, many studies illustrate old paternal age and its disadvantageous impacts on sperm quality and parameter [2]. In one study about sperm count and sperm motility in old rats, it has been found that sperm count was fallen notably in rat female's uterus at 24 months of age, due to a reduction in sperm production. Also, the prostate gland and dorsal prostatic secretions, which are required for sperm motility, had morphological prostate alterations and secretion modification at aging rats Also, the study results showed a link between sperm immobility and motility and a failure in the prostatic function in old male rats, when sperm motility was decreased at both 12 months, 24 months of age (Lucio., et al. 2013) (Figure 2).

Moreover, any medicine, tumour, illness, or disorder that influences the pituitary gland or hypothalamus could be a potential cause of male infertility through modifying gonadotropic releasing hormone or producing gonadotropin as idiopathic hypogonadotropic hypogonadism (IHH), Kallmann syndrome (IHH with anosmia), and mixed pituitary hormone deficiency (Leslie SW., et al. 2020) [3]. In addition, pituitary tumours like sellar tumours, macroadenomas, and prolactinomas can also rise in male infertility due to changes in gonadotropin hormone production and Besides, sev-

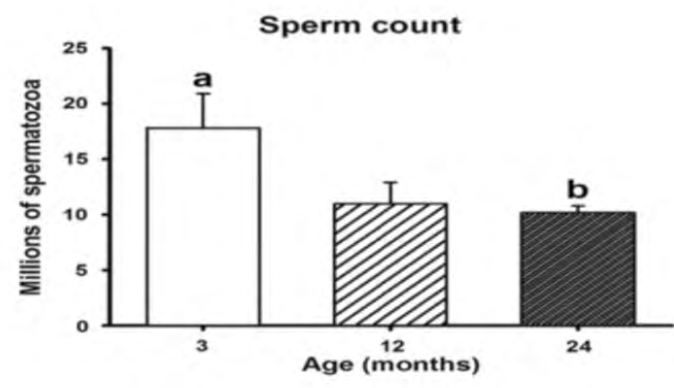

Figure 2: Sperm count alteration in aging rat; the diagram illustrate that sperm count is decaling with increasing rat age (Lucio., et al. 2013).

eral genetic causes Prader-Willi, Young, and Laurence-Moon-Biedl syndromes, can lead to male infertility. Many acquired disorders, such as primary androgen overproduction and exogenous testosterone supplementation, would also instantly reduce gonadotropic secretion inducing decreased sperm counts and infertility (Leslie SW,., et al. 2020).

\section{Semen analysis and male infertility}

Semen has two main elements: the spermatozoa produced by the testis' seminiferous tubules. The seminal fluid made at the accessory glands supports sperm and has a premium role in interacting with the female reproductive tract to induce fertilization. These elements are shown in the semen analysis through the sperm count, that indicates the number of spermatozoa in the semen sample; and the volume of the semen, which reveals the amount of seminal fluid produced. Sperm motility is described as the rate of sperm that moves through the seminal fluid, while the sperm morphology is the percentage of sperm with a standard cellular structure (Patel, Leong and Ramasamy, 2018) [4]. For accurate results, the semen sample is obtained by masturbation after an abstinence period of 2-7 days. The semen sample has some physical characteristics which can be measured, like the volume, $\mathrm{pH}$, colour, liquefaction, and viscosity. The sample is then assessed through a microscope to define the motility, vitality, concentration, and morphology. The semen analysis results received are compared to the reference values determined by the WHO manual (Patel, Leong and Ramasamy, 2018). 


\section{Abnormal semen sample}

The semen analysis can indicate several and various semen sample abnormalities. The abnormalities can be related to concentration, morphology, and motility of the sperms (Table 1). For instance, Azoospermia is a condition where spermatozoa is absence in the sediment of a centrifuged semen sample of a man', where cryptozoospermia is an abnormal semen condition defined as 'very low spermatozoa concentration ( $\leq 1 \mathrm{million} / \mathrm{mL}$ ) in the ejaculate of a man'. These circumstances are usually diagnosed through a routine male infertility examination.

\begin{tabular}{|l|c|}
\hline \multicolumn{1}{|c|}{ Term } & Definitions \\
\hline Normozoospermia & $\begin{array}{c}\text { Normal ejaculate as defined by the } \\
\text { reference values }\end{array}$ \\
\hline Oligozoospermia & $\begin{array}{c}\text { Sperm concentration fewer than } 20 \\
\mathrm{x} 10^{\wedge} 6 / \mathrm{mL}\end{array}$ \\
\hline Asthenozoospermia & $\begin{array}{c}\text { Fewer than } 50 \% \text { spermatozoa with } \\
\text { forward progression (categories 'a' } \\
\text { and 'b') or fewer than 25\% } \\
\text { spermatozoa with category 'a' } \\
\text { movement }\end{array}$ \\
\hline Teratozoospermia & $\begin{array}{c}\text { Fewer than 30\% spermatozoa with } \\
\text { normal morphology Less than the } \\
\text { reference value for morphology }\end{array}$ \\
\hline $\begin{array}{l}\text { Oligoasthenoterato- } \\
\text { zoospermia }\end{array}$ & $\begin{array}{c}\text { Signifies disturbance of all three } \\
\text { variables (combinations of only two } \\
\text { prefixes may also be used) }\end{array}$ \\
\hline Azoospermia & No spermatozoa in the ejaculate \\
\hline Aspermia & No ejaculate \\
\hline
\end{tabular}

Table 1: The WHO categorization of an abnormal semen sample (WHO, 2010).

As discussed above, Cryptozoospermia (CO) is a condition described by the World Health Organization where spermatozoa cannot be recognized or found in a fresh semen sample. Still, it can be observed with extended centrifugation and microscopic process. However, the spermatozoa found in a section of semen samples using a microscopic directly, but cannot be included due to low concentration; this condition is diagnosed as a severe oligozoospermia (ESO) (Deng., et al. 2015) [5]. ESO that has not been described by the World Health Organization is explicitly a state that spermatozoa concentration is $<1 \times 106 \mathrm{ml}-1$, based on the definition of severe oligoasthenoteratozoospermia by various studies. ESO and CO patients are advised to go for intracytoplasmic sperm injection (ICSI) as infertility treatment as their partners are challenging to get pregnant easily or by drug treatment. ICSI assists them to be biological parents by using their single spermatozoa that have been collected from ejaculation or percutaneous epididymal sperm aspiration (PESA) or testicular sperm aspiration (TESA), even testicular sperm extraction (TESE) or micro-TESE.CO can be influenced by environment temperature, the atmosphere of the masturbation room, the ejaculation state, induction by audio-visual devices, etc. In cryptozoospermia, the sperm count fluctuates remarkably in sperm count, pointing to challenges in obtaining sperm in the ejaculate on the day of oocyte retrieval (Koscinski., et al. 2013). Thus CO is usually thought to be virtual azoospermia and regularly needs testicular sperm extraction (TESE) for intracytoplasmic sperm injection (ICSI) treatment. Additionally, CO can cause various risks to male infertility treatment; for instance, possible andrological risks are linked to testicular biopsies, such as vascular injuries and additional androgenic defect or testicular atrophy; the utility of testicular gametes, that can be less mature from the ejaculated spermatozoa. Therefore, an extended sperm preparation (ESP) protocol should be applied regularly to confirm the accurate diagnostic of $\mathrm{CO}$ and azoospermia, particularly in the absence of obstructive aetiology (Koscinski., et al. 2013) [6]. On the other hand, oligozoospermia is frequently linked with exogenous factors like infectious agents, environmental pollutants, age, and obesity. Male with oligozoospermia does have few sperm, more than those with azoospermia or $\mathrm{CO}$, although significantly fewer than normal fertile male. Furthermore, this condition is associated with irregular sperms morphology (Badal, 2017). When oligozoospermia developed to a severe case, its then called severe oligozoospermia (ESO), and its equal to Cryptozoospermia. Both CO and ESO can be caused by genetic abnormalities, medical history and abnormal physical examination. For instance, an abnormal karyotype in 47,XXY and hypogonadism can cause CO; also deletion of the azoospermia factor (AZF) region and hypogonadism can also cause $\mathrm{CO}$ and azoospermia (Dohle, 2002). Still, ESO and CO are not etiologist representative, but only an analysis of semen parameters. Hence, in some cases, patients can have testicular dysfunction like Y chromosome microdeletion, and others may have incomplete seminal tract obstruction conditions. At the same time, their spermatogenesis is normal as OA. This can create heterogeneity of patients in 
ESO and CO Groups .In another case study, 33- years old patient, who had go for sterility treatment for almost 5 years. Through the diagnostic journey, a novel homozygous nonsense mutation in has been found, which was associated with cryptozoospermia. This mutation can also be a genetic factor of cryptozoospermia, with TEX15 deficiency causing spermatogenesis failure [6]. The mutation was inherited from the patient's parents, who were both heterozygous carriers, representative a recessive pattern of inheritance, with the development of an effective medicine, will make TEX15 deficiency to become a clinical marker for recognition of nonobstructive azoospermia or cryptozoospermia (Sha., et al. 2018). The treatment main treatment option for male infertility in cases of CO and ESO is ICSI. ICSI was a transformation method which was capable to avoid difficulties in natural sperm and oocyte fertilization and fusion, particularly appropriate for sever oligozoospemia and cryptozoospermia. Although, if no sperm can be obtained on the day of oocyte retrieval it can leads to the cancelation of ICSI (Deng., et al. 2015). Thus, the testis biopsy can be the gold standard in such cases to assess spermatogenesis, which is not a routine investigation in CO and ESO. Consequently, it is essential to evaluate spermatogenic functions in patients with $\mathrm{CO}$ and ESO, through volume of testis size, levels of FSH and inhibin B in advance. Additionally, it is prefeed to obtain a testicular if CO and ESO was accompanied with azoospermia (Deng., et al. 2015). Additionally, To avoid ICSI cancellation on the day of oocytes retrieval, most IVF centres offer a substitute options such as surgically sperm extraction (PESA or TESE) to synchronize oocyte retrieval (Ketabchi, 2016) [7,8].

\section{Cryptozoospermia and cryopreservation}

Cryopreservation of human sperms has been applied in the 1960 's, where it has been known to be an effective method of the male fertility management before treatment for malignant diseases, vasectomy or surgical infertility treatments, to save donor and partner spermatozoa before assisted reproduction procedures and to assure the recovery of a small number of spermatozoa in severe male factor infertility, such as in the cases of the cryptozoospermia or sever oligospermia (Di Santo, Tarozzi, Nadalini and Borini, 2012) [9]. The traditional approaches of sperm cryopreservation cannot be applied to freeze small numbers of cells, like epididymal and testicular spermatozoa. Thus an effective and novel cryopreservation of surgically gained spermatozoa, can decreases the number of surgical interventions and reduced the logistic risks as- sociated with managing the women's oocyte retrieval, additionally to the risk of no sperm can be gained on the same day of oocyte retrieval (Di Santo, Tarozzi, Nadalini and Borini, 2012). Conventional sperm cryopreservation methods can lead to sperm loss through sperm adherence to the carrier vessel, inadequate centrifugation, and washing phase. Therefore, the traditional approach is especially problematic in cases such as severe oligospermia and cryptozoospermia (Liu and Li, 2020) [10]. A novel cryopreservation method for few sperms utilises an empty zona pellucida (ZP) by providing theoretical and technical support for other procedures. After introducing such a single-sperm freezing technique, several cryopreservation methods have been introduced to enhance sperm counts (Liu and Li, 2020). Similar to the non-biological empty ZP method, an empty capsule of a non-biological carrier has been developed which is received from an animal. Where small spermatozoa have been frozen in polymerized alginic acid drops. Alginic acid is an inert chemical of two sugars mixture, namely, $\beta$-D-mannuronic and $\alpha$-L-guluronic acid. Alginate capsules carrying sperm are stored in the cryoprotective agent (CPA) and preserved in straws using a programmable freezer. Upon thawing, alginate capsules are dissolved in a sodium citrate solution [11]. The advantages of using alginate is that it is a non-hazardous polysaccharide, it can be applied successfully for the cryopreservation of hepatocytes and stem cells, it has gel liquid characteristics and its inertness chemical. However, cryopreservation of human sperm through this procedure produced in a $20 \%$ reduction in motility compared with standard protocols. It is proposed that this is possibly caused by the alginic acid covering the surface of the sperm. Owing to the complexity of the complete operation and other shortcomings, this technology's developments are slow, thus limiting its clinical application (Liu and Li, 2020). Another effective method applied for cryptozoospermia cryopreservation is called cell sleeper, which is a closed cryopreservation system. The Cell Sleeper has a vial container implemented with an internal tray, where individual sperm can be added to a droplet $(3.5 \mu \mathrm{L})$ on the tray. Following, the tray is placed into a vial and covered by a screw cap. The vial is then put in LN2 vapour $\left(-120^{\circ} \mathrm{C}\right)$ before the exposure to sterilized LN2 [13].

\section{Conclusion}

In conclusion, male infertility is common and can account for a high percentage of infertility disorder. CO and ESO are complex male infertility disorder, which can be caused by several factors 
such as genetics and hormonal factors. Therefore, sperms left in the male reproductive system in $\mathrm{CO}$ and ESO cases can be the last hope key for numerous partners; thus, they need to be collected and treated in a well organised and effective way [13]. Cryopreservation would be the best $\mathrm{CO}$ and ESO options to preserve individual sperms; still, a well-developed protocol and techniques should be used to protect those individual sperms.

\section{Bibliography}

1. Babakhanzadeh E., et al. "Some of the Factors Involved in Male Infertility: A Prospective Review". International Journal of General Medicine 13 (2020): 29-41.

2. Bakare T., et al. "Cryptozoospermia". Fertility and Sterility 108.3 (2017): e313-e314.

3. Deng Y., et al. "Differences and Similarities between extremely severe oligozoospermia and cryptozoospermia in intracytoplasmic sperm injection". Asian Journal of Andrology 18 (2015): 904-907.

4. Dohle G. "Genetic risk factors in infertile men with severe oligozoospermia and azoospermia". Human Reproduction 17.1 (2002): 13-16.

5. Ketabchi A. "Intracytoplasmic Sperm Injection Outcomes with Freshly Ejaculated Sperms and Testicular or Epididymal Sperm Extraction in Patients with Idiopathic Cryptozoospermia”. Nephro-Urology Monthly 8.6 (2016).

6. Koscinski I., et al. "Pregnancies in Cryptozoospermia With Sperm Ejaculated One Day Before ICSI: Four Case Reports". Journal of Andrology 28.1 (2013): 15-20.

7. Leslie SW., et al. "Male Infertility". Treasure Island (FL): StatPearls Publishing (2020).

8. Liu S and Li F. "Cryopreservation of single-sperm: where are we today?". Reproductive Biology and Endocrinology 18.1 (2020).

9. Lucio R., et al. "Sperm count and sperm motility decrease in old rats". Physiology and Behavior 110-111 (2013): 73-79.

10. Miyamoto T., et al. "Male infertility and its causes in human". Advances in Urology (2012): 384520.
11. Patel A., et al. "Prediction of male infertility by the World Health Organization laboratory manual for assessment of semen analysis: A systematic review". Arab Journal of Urology 16.1 (2018): 96-102.

12. Sha Y., et al. "Case study of a patient with cryptozoospermia associated with a recessive TEX15 nonsense mutation". Asian Journal of Andrology 20.1 (2018): 101.

13. Sharma R., et al. "Effects of increased paternal age on sperm quality, reproductive outcome and associated epigenetic risks to offspring". Reproductive Biology and Endocrinology (2015).

\section{Assets from publication with us}

- Prompt Acknowledgement after receiving the article

- Thorough Double blinded peer review

- Rapid Publication

- Issue of Publication Certificate

- High visibility of your Published work

Website: www.actascientific.com/

Submit Article: www.actascientific.com/submission.php

Email us: editor@actascientific.com

Contact us: +919182824667 